\title{
EnlightenGAN: Deep Light Enhancement without Paired Supervision
}

\author{
Yifan Jiang, Xinyu Gong, and Ding Liu, Yu Cheng, Chen Fang, Xiaohui Shen, \\ Jianchao Yang, Pan Zhou, and Zhangyang Wang
}

\begin{abstract}
Deep learning-based methods have achieved remarkable success in image restoration and enhancement, but are they still competitive when there is a lack of paired training data? As one such example, this paper explores the low-light image enhancement problem, where in practice it is extremely challenging to simultaneously take a low-light and a normal-light photo of the same visual scene. We propose a highly effective unsupervised generative adversarial network, dubbed EnlightenGAN, that can be trained without low/normal-light image pairs, yet proves to generalize very well on various real-world test images. Instead of supervising the learning using ground truth data, we propose to regularize the unpaired training using the information extracted from the input itself, and benchmark a series of innovations for the low-light image enhancement problem, including a global-local discriminator structure, a selfregularized perceptual loss fusion, and the attention mechanism. Through extensive experiments, our proposed approach outperforms recent methods under a variety of metrics in terms of visual quality and subjective user study. Thanks to the great flexibility brought by unpaired training, EnlightenGAN is demonstrated to be easily adaptable to enhancing real-world images from various domains. Our codes and pre-trained models are available at: https://github.com/VITA-Group/EnlightenGAN.
\end{abstract}

Index Terms-Low-light Enhancement, Generative Adversarial Networks, Unsupervised Learning.

\section{INTRODUCTION}

$\mathbf{I}$ MAGE captured in low-light conditions suffer from low contrast, poor visibility and high ISO noise. Those issues challenge both human visual perception that prefers highvisibility images, and numerous intelligent systems relying on computer vision algorithms such as all-day autonomous driving and biometric recognition [1]. To mitigate the degradation, a large number of algorithms have been proposed, ranging from histogram or cognition-based ones [2], [3] to learningbased approaches [4], [5]. The state-of-the-art image restoration and enhancement approaches using deep learning heavily rely on either synthesized or captured corrupted and clean image pairs to train, such as super-resolution [6], denoising [7] and deblurring [8].

Y. Jiang, X. Gong and Z. Wang are with the Department of Electrical and Computer Engineering, the University of Texas at Austin, TX, USA. (email: yifanjiang97@utexas.edu; xinyu.gong@utexas.edu; atlaswang@utexas.edu)

D. Liu, C. Fang, X. Shen and J. Yang are with Bytedance Inc. (email: liuding@bytedance.com; fangchen@bytedance.com; shenxiaohui.kevin@bytedance.com; yangjianchao@bytedance.com)

Y. Cheng is with Microsoft AI \& Research, Redmond, Washington 98052, USA. (e-mail: yu.cheng@microsoft.com)

P. Zhou is with Department of Electronic Information and Communication, Huazhong University of Science and Technology. (email: panzhou@ hust.edu.cn)

Correspondence addressed to: Zhangyang Wang (atlaswang@utexas.edu)
However, the availability assumption of paired training images has raised more difficulties, when it comes to enhancing images from more uncontrolled scenarios, such as dehazing, deraining or low-light enhancement: 1) it is very difficult or even impractical to simultaneously capture corrupted and ground truth images of the same visual scene (e.g., low-light and normal-light image pairs at the same time);2) synthesizing corrupted images from clean images could sometimes help, but such synthesized results are usually not photo-realistic enough, leading to various artifacts when the trained model is applied to real-world low-light images; 3 ) specifically for the low-light enhancement problem, there may be no unique or well-defined high-light ground truth given a low-light image. For example, any photo taken from dawn to dusk could be viewed as a highlight version for the photo taken over the midnight at the same scene. Taking into account the above issues, our overarching goal is to enhance a low-light photo with spatially varying light conditions and over/under-exposure artifacts, while the paired training data is unavailable.

Inspired by [9], [10] for unsupervised image-to-image translation, we adopt generative adversarial networks (GANs) to build an unpaired mapping between low and normal light image spaces without relying on exactly paired images. That frees us from training with only synthetic data or limited real paired data captured in controlled settings. We introduce a lightweight yet effective one-path GAN named EnlightenGAN, without using cycle-consistency as prior works [11], [12], [13], [14] and therefore enjoying the merit of much shorter training time.

Due to the lack of paired training data, we incorporate a number of innovative techniques. We first propose a dualdiscriminator to balance global and local low-light enhancement. Further, owing to the absence of ground-truth supervision, a self-regularized perceptual loss is proposed to constrain the feature distance between the low-light input image and its enhanced version, which is subsequently adopted both locally and globally together with the adversarial loss for training EnlightenGAN. We also propose to exploit the illumination information of the low-light input as a self-regularized attentional map in each level of deep features to regularize the unsupervised learning. Thanks to the unsupervised setting, we show that EnlightenGAN can be very easily adapted to enhancing real-world low-light images from different domains.

We highlight the notable innovations of EnlightenGAN:

- EnlightenGAN is the first work that successfully introduces unpaired training to low-light image enhancement. Such a training strategy removes the dependency on 

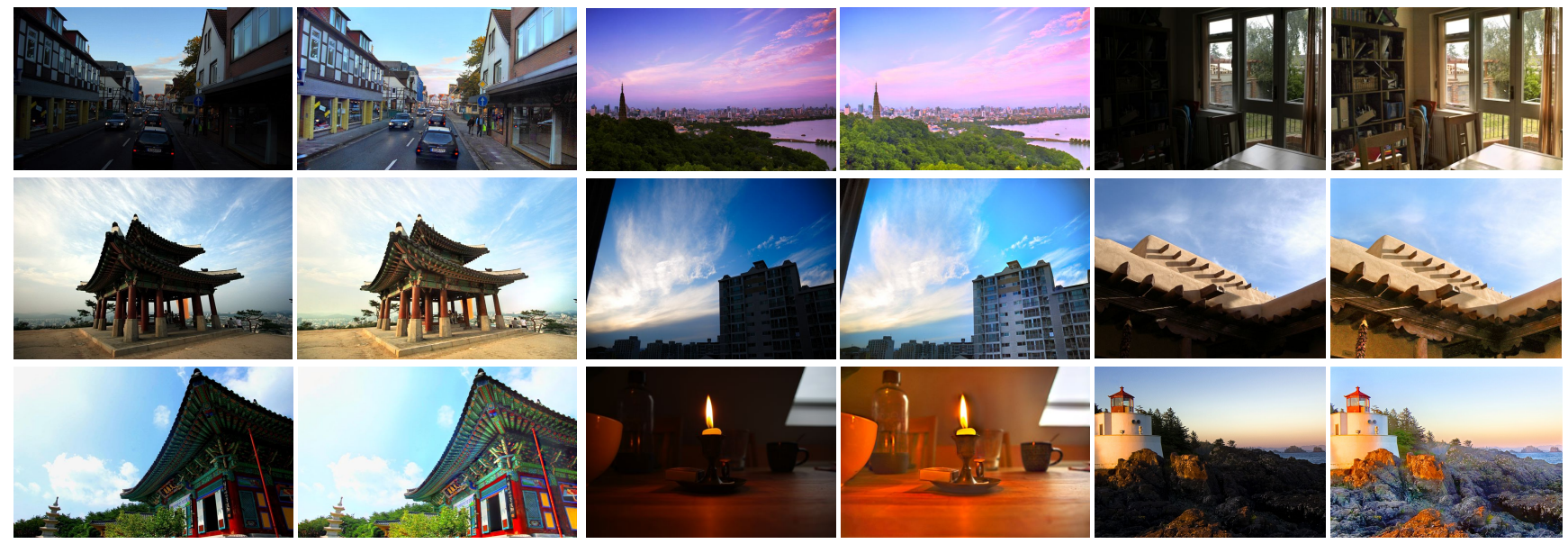

Fig. 1: Representative visual examples by enhancing low-light images using EnlightenGAN. From left to right: columns 1, 3 and 5 are the low-light input images; while columns 2, 4, an 6 their corresponding enhanced images by EnlightenGAN.

paired training data and enables us to train with larger varieties of images from different domains. It also avoids overfitting any specific data generation protocol or imaging device that previous works [15], [5], [16] implicitly rely on, hence leading to notably improved real-world generalization.

- EnlightenGAN gains remarkable performance by imposing (i) a global-local discriminator structure that handles spatially-varying light conditions in the input image; (ii) the idea of self-regularization, implemented by both the self feature preserving loss and the self-regularized attention mechanism. The self-regularization is critical to our model success, because of the unpaired setting where no strong form of external supervision is available.

- EnlightenGAN is compared with several state-of-theart methods via comprehensive experiments. The results are measured in terms of visual quality, no-referenced image quality assessment, and human subjective survey. All results consistently endorse the superiority of EnlightenGAN. Moreover, in contrast to existing pairedtrained enhancement approaches, EnlightenGAN proves particularly easy and flexible to be adapted to enhancing real-world low-light images from different domains.

\section{RELATED WORKS}

Paired Datasets: Status Quo. There exist several options to collect a paired dataset of low/normal-light images, but unfortunately none is efficient nor easily scalable. One may fix a camera and then reduce the exposure time in normallight condition [5] or increase exposure time in low-light condition [16]. The LOL dataset [5] is so far the only dataset of low/normal-light image pairs taken from real scenes by changing exposure time and ISO. Due to the tedious experimental setup, e.g. the camera needs to be fixed and the object cannot move, etc., it consists of only 500 pairs. Moreover, it may still deviate from the true mapping between natural low/normallight images. Especially under spatially varying lights, simply increasing/decreasing exposure time may lead to local over/under-exposure artifacts.

In the high-dynamic-ranging (HDR) field, a few works first capture several images at different imperfect light conditions, then align and fuse them into one high-quality image [15], [17]. However, they are not designed for the purpose of postprocessing only one single low-light image.

Traditional Approaches. Low-light image image enhancement has been actively studied as an image processing problem for long, with a few classical methods such as the adaptive histogram equalization (AHE) [3], Retinex [2] and multiscale Retinex model [18]. More recently, [19] proposed an enhancement algorithm for non-uniform illumination images, utilizing a bi-log transformation to make a balance between details and naturalness. Based on the previous investigation of the logarithmic transformation, Fu et al. proposed a weighted variational model [20] to estimate both the reflectance and the illumination from an observed image with imposed regularization terms. In [21], a simple yet effective low-light image enhancement (LIME) was proposed, where the illumination of each pixel was first estimated by finding the maximum value in its RGB channels, then the illumination map was constructed by imposing a structure prior. [22] introduced a joint low-light image enhancement and denoising model via decomposition in a successive image sequence. [23] further proposed a robust Retinex model, which additionally considered a noise map compared with the conventional Retinex model, to improve the performance of enhancing low-light images accompanied by intensive noise.

Deep Learning Approaches. Existing deep learning solutions mostly rely on paired training, where most low-light images are synthesized from normal images. [4] proposed a stacked auto-encoder (LL-Net) to learn joint denoising and low-light enhancement on the patch level. Retinex-Net in [5] provided an end-to-end framework to combine the Retinex theory and deep networks. HDR-Net [24] incorporated deep networks with the ideas of bilateral grid processing and local affine color transforms with pairwise supervision. A few multi-frame low-light enhancement methods were developed in the HDR 


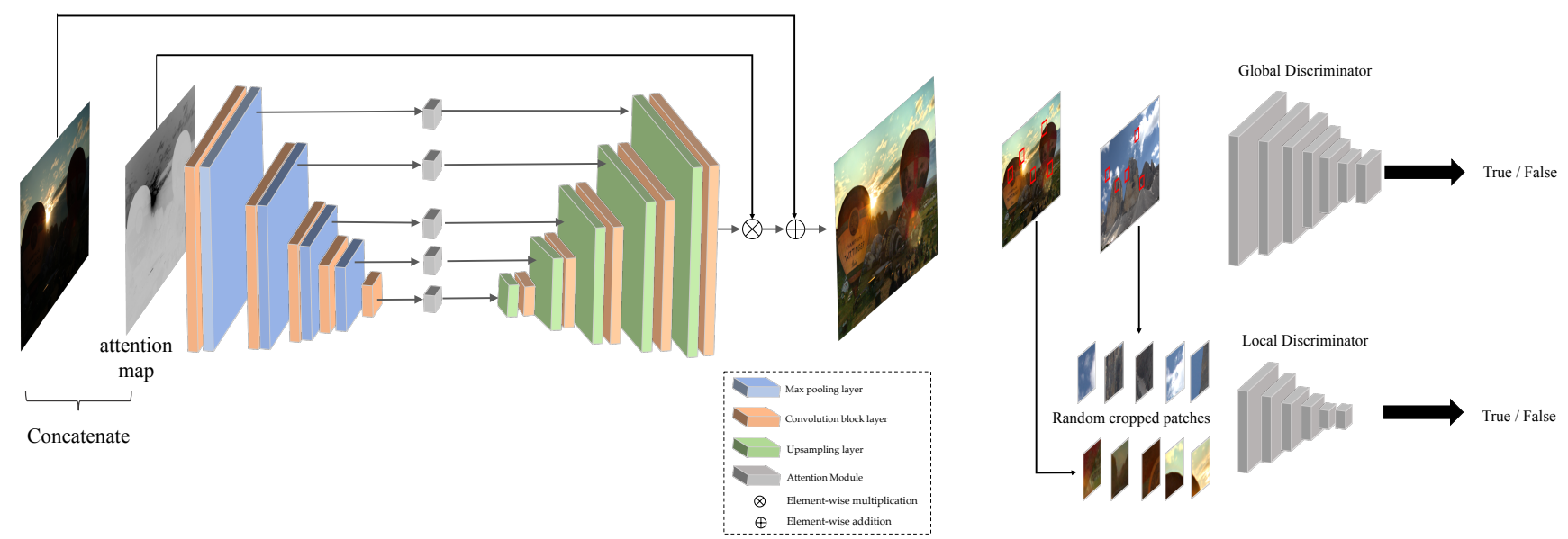

Fig. 2: The overall architecture of EnlightenGAN. In the generator, each convolutional block consists of two $3 \times 3$ convolutional layers followed by batch normalization and LeakyRelu. Each attention module has the feature map multiply with a (resized) attention map.

domain, such as [15], [17], [25].

Lately, [16] proposed a "learning to see in the dark" model that achieves impressive visual results. However, this method operates directly on raw sensor data, in addition to the requirement of paired low/normal-light training images. Besides, it focuses more on avoiding the amplified artifacts during low-light enhancement by learning the pipeline of color transformations, demosaicing and denoising, which differs from EnlightenGAN in terms of settings and goal.

Adversarial Learning. GANs [26], [27] have proven successful in image synthesis and translation. When applying GANs to image restoration and enhancement, most existing works use paired training data as well, such as super resolution [28], artistic style transfer and image editing [29], [30], deraining [31] and dehazing [32]. Several unsupervised GANs are proposed to learn inter-domain mappings using adversarial learning and are adopted for many other tasks. [9], [10] adopted a two-way GAN to translate between two different domains by using a cycle-consistent loss with unpaired data A handful of latest works followed their methodology and applied unpaired training with cycle-consistency to several lowlevel vision tasks, e.g. dehazing, deraining, super-resolution and mobile photo enhancement [33], [34], [35], [36]. Different from them, EnlightenGAN refers to unpaired training but with a lightweight one-path GAN structure (i.e., without cycleconsistency), which is stable and easy to train.

\section{Method}

As shown in Fig. 2, our proposed method adopts an attention-guided U-Net as the generator and uses the dualdiscriminator to direct the global and local information. We also use a self feature preserving loss to guide the training process and maintain the textures and structures. In this section we first introduce two important building blocks, i.e., the global-local discriminators and the self feature preserving loss, then the whole network in details. The detailed network architectures are in the supplementary materials.

\section{A. Global-Local Discriminators}

We adopt the adversarial loss to minimize the distance between the real and output normal light distributions. However, we observe that an image-level vanilla discriminator often fails on spatially-varying light images; if the input image has some local area that needs to be enhanced differently from other parts, e.g., a small bright region in an overall dark background, the global image discriminator alone is often unable to provide the desired adaptivity.

Inspired by previous work [37], to enhance local regions adaptively in addition to improving the light globally, we propose a novel global-local discriminator structure, both using PatchGAN for real/fake discrimination. In addition to the image-level global discriminator, we add a local discriminator by taking randomly cropped local patches from both output and real normal-light images, and learning to distinguish whether they are real (from real images) or fake (from enhanced outputs). Such a global-local structure ensures all local patches of an enhanced images look like realistic normal-light ones, which proves to be critical in avoiding local over- or under-exposures as our experiments will reveal later.

Furthermore, for the global discriminator, we utilize the recently proposed relativistic discriminator structure [38] which estimates the probability that real data is more realistic than fake data and also directs the generator to synthesize a fake image that is more realistic than real images. The standard function of relativistic discriminator is:

$$
\begin{aligned}
& D_{R a}\left(x_{r}, x_{f}\right)=\sigma\left(C\left(x_{r}\right)-\mathbb{E}_{x_{f} \sim \mathbb{P}_{\text {fake }}}\left[C\left(x_{f}\right)\right]\right), \\
& D_{R a}\left(x_{f}, x_{r}\right)=\sigma\left(C\left(x_{f}\right)-\mathbb{E}_{x_{r} \sim \mathbb{P}_{\text {real }}}\left[C\left(x_{r}\right)\right]\right),
\end{aligned}
$$

where $C$ denotes the network of discriminator, $x_{r}$ and $x_{f}$ are sampled from the real and fake distribution, $\sigma$ represents the sigmoid function. We slight modify the relativistic discriminator to replace the sigmoid function with the least-square GAN 


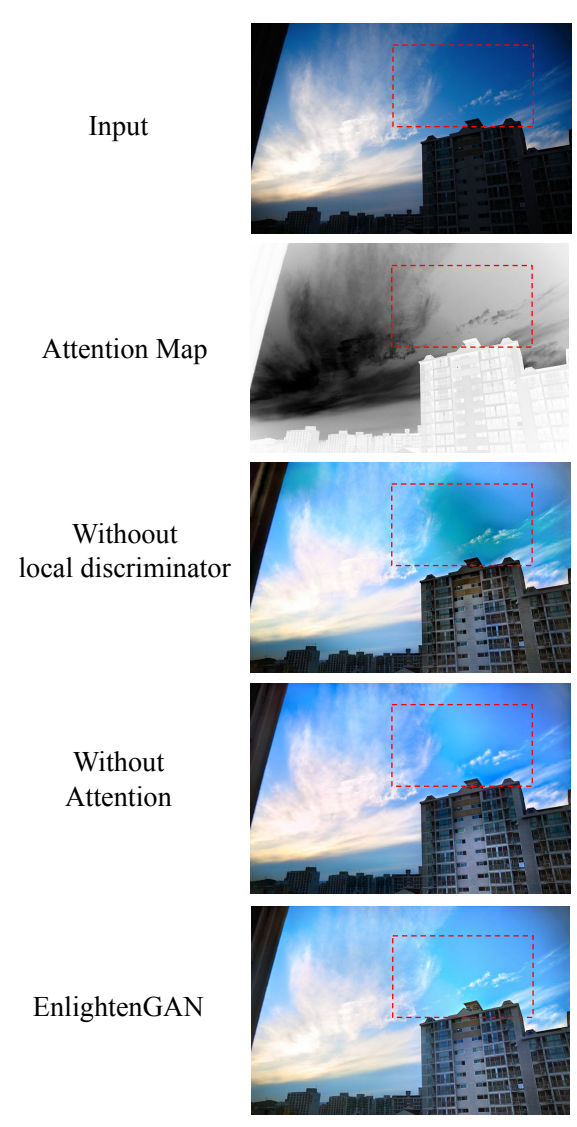

(a)
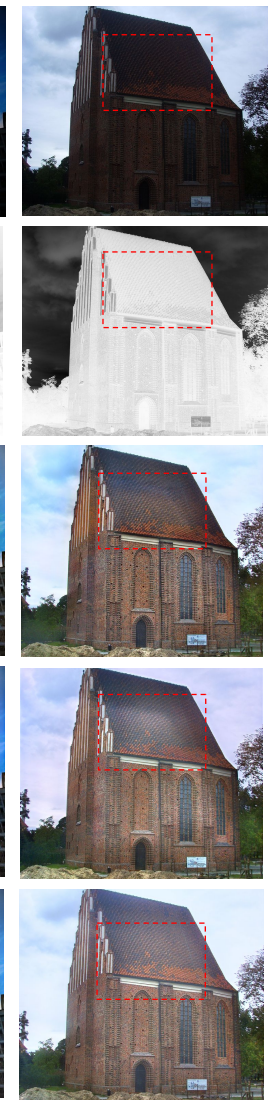

(b)

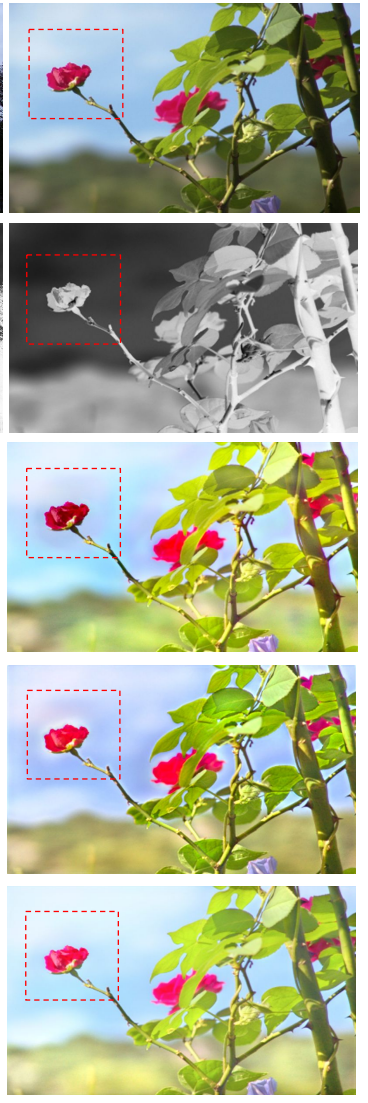

(c)
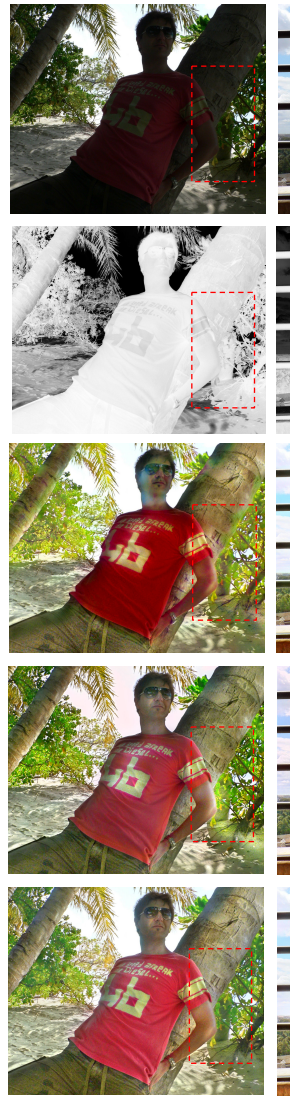

(d)
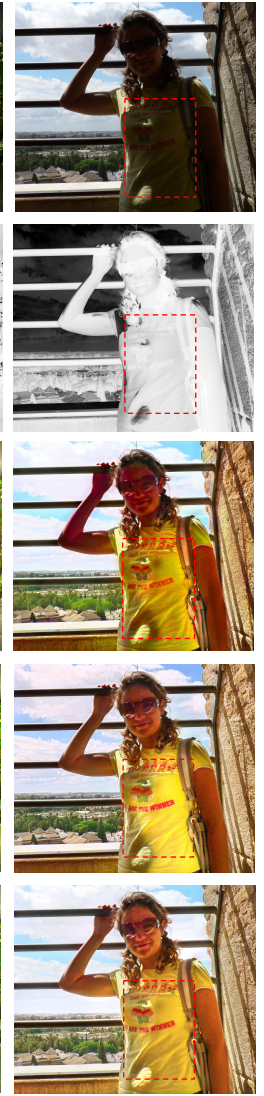

(e)

Fig. 3: Visual comparison from the ablation study of EnlightenGAN. Row $1 \sim 5$ display the low-light image inputs, the attention map of input, results from EnlightenGAN with only global discriminator, results from EnlightenGAN without self-regularized attention mechanism, and results from the final version of EnlightenGAN, respectively. Images in Row 3 and 4 suffer from severe color distortion or inconsistency, which are highlighted by bounding boxes. The final version of EnlightenGAN is able to mitigate the above issues and gains the most visually pleasing results. Please zoom in to see the details.

(LSGAN) [39] loss. Finally, the loss functions for the global discriminator $D$ and the generator $G$ are:

$$
\begin{aligned}
\mathcal{L}_{D}^{\text {Global }}=\mathbb{E}_{x_{r} \sim \mathbb{P}_{\text {real }}}\left[\left(D_{R a}(\right.\right. & \left.\left.\left.x_{r}, x_{f}\right)-1\right)^{2}\right] \\
& +\mathbb{E}_{x_{f} \sim \mathbb{P}_{\text {fake }}}\left[D_{R a}\left(x_{f}, x_{r}\right)^{2}\right], \\
\mathcal{L}_{G}^{\text {Global }}=\mathbb{E}_{x_{f} \sim \mathbb{P}_{\text {fake }}}\left[\left(D_{R a}(\right.\right. & \left.\left.\left.x_{f}, x_{r}\right)-1\right)^{2}\right] \\
& +\mathbb{E}_{x_{r} \sim \mathbb{P}_{\text {real }}}\left[D_{R a}\left(x_{r}, x_{f}\right)^{2}\right],
\end{aligned}
$$

For the local discriminator, we randomly crop 5 patches from the output and real images each time. Here we adopt the original LSGAN as the adversarial loss, as follows:

$$
\begin{aligned}
& \mathcal{L}_{D}^{\text {Local }}=\mathbb{E}_{x_{r} \sim \mathbb{P}_{\text {real-patches }}} {\left[\left(D\left(x_{r}\right)-1\right)^{2}\right] } \\
&+\mathbb{E}_{x_{f} \sim \mathbb{P}_{\text {fake-patches }}}\left[\left(D\left(x_{f}\right)-0\right)^{2}\right] \\
& \mathcal{L}_{G}^{L o c a l}=\mathbb{E}_{x_{r} \sim \mathbb{P}_{\text {fake-patches }}}\left[\left(D\left(x_{f}\right)-1\right)^{2}\right]
\end{aligned}
$$

\section{B. Self Feature Preserving Loss}

To constrain the perceptual similarity, Johnson et al. [40] proposed perceptual loss by adopting a pre-trained VGG to model feature space distance between images, which was widely adopted to many low-level vision tasks [28], [41]. The common practice constrains the extracted feature distance between the output image and its ground truth.

In our unpaired setting, we propose to instead constrain the VGG-feature distance between the input low-light and its enhanced normal-light output. This is based on our empirical observation that the classification results by VGG models are not very sensitive when we manipulate the input pixel intensity range, which is concurred by another recent study [42]. We call it self feature preserving loss to stress its self-regularization utility to preserve the image content features to itself, before and after the enhancement. That is distinct from the typical usage of the perceptual loss in (paired) image restoration, and is motivated from our unpaired setting too. Concretely, the self feature preserving loss $L_{S F P}$ is defined as:

$$
\mathcal{L}_{S F P}\left(I^{L}\right)=\frac{1}{W_{i, j} H_{i, j}} \sum_{x=1}^{W_{i, j}} \sum_{y=1}^{H_{i, j}}\left(\phi_{i, j}\left(I^{L}\right)-\phi_{i, j}\left(G\left(I^{L}\right)\right)\right)^{2}
$$

where $I^{L}$ denotes the input low-light image and $G\left(I^{L}\right)$ denotes the generator's enhanced output. $\phi_{i, j}$ denotes the feature 

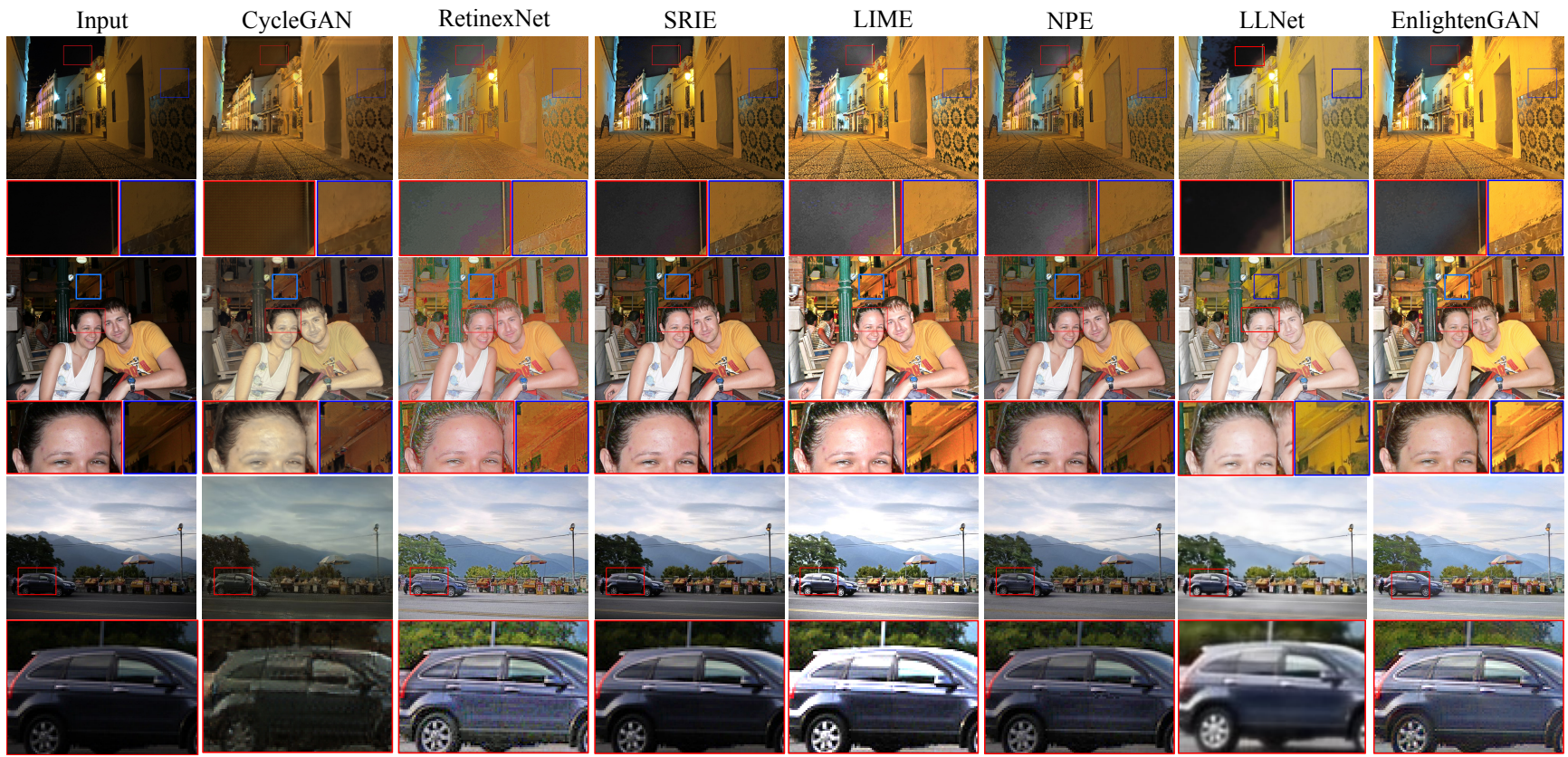

Fig. 4: Comparison with other state-of-the-art methods. Zoom-in regions are used to illustrate the visual differences. Three examples are listed from the top to the bottom rows. First example: EnlightenGAN successfully suppresses the noise in black sky and produces the best visible details of yellow wall. Second example: NPE and SRIE fail to enhance the background details. LIME introduces over-exposure on the woman's face. LLNet generate severe color distortion. However, EnlightenGAN not only restores the background details but also avoids over-exposure artifacts, distinctly outperforming other methods. Third example: EnlightenGAN produces a visually pleasing result while avoiding over-exposure artifacts in the car and cloud. Others either do not enhance dark details enough or generate over-exposure artifacts. Please zoom in to see more details.

map extracted from a VGG-16 model pre-trained on ImageNet. $i$ represents its $i$-th max pooling, and $j$ represents its $j$-th convolutional layer after $i$-th max pooling layer. $W_{i, j}$ and $H_{i, j}$ are the dimensions of the extracted feature maps. By default we choose $i=5, j=1$.

For our local discriminator, the cropped local patches from input and output images are also regularized by a similarly defined self feature preserving loss, $L_{S F P}^{\text {Local }}$. Furthermore, We add an instance normalization layer [43] after the VGG feature maps before feeding into $L_{S F P}$ and $L_{S F P}^{L o c a l}$ in order to stabilize training. The overall loss function for training EnlightenGAN is thus written as:

$$
\text { Loss }=\mathcal{L}_{S F P}^{\text {Global }}+\mathcal{L}_{S F P}^{\text {Local }}+\mathcal{L}_{G}^{\text {Global }}+\mathcal{L}_{G}^{\text {Local }},
$$

\section{U-Net Generator Guided with Self-Regularized Attention}

U-Net [44] has achieved huge success on semantic segmentation, image restoration and enhancement [45]. By extracting multi-level features from different depth layers, U-Net preserves rich texture information and synthesizes high quality images using multi-scale context information. We adopt UNet as our generator backbone.

We further propose an easy-to-use attention mechanism for the U-Net generator. Intuitively, in a low-light image of spatially varying light condition, we always want to enhance the dark regions more than bright regions, so that the output image has neither over- nor under-exposure. We take the illumination channel $I$ of the input RGB image, normalize it to
$[0,1]$, and then use $1-I$ (element-wise difference) as our selfregularized attention map. We then resize the attention map to fit each feature map and multiply it with all intermediate feature maps as well as the output image. We emphasize that our attention map is also a form of self-regularization, rather than learned with supervision. Despite its simplicity, the attention guidance shows to improve the visual quality consistently.

Our attention-guided U-Net generator is implemented with 8 convolutional blocks. Each block consists of two $3 \times 3$ convolutional layers, followed by LeakyReLu and a batch normalization layer [46]. At the upsampling stage, we replace the standard deconvolutional layer with one bilinear upsampling layer plus one convolutional layer, to mitigate the checkerboard artifacts. The final architecture of EnlightenGAN is illustrated in the left of Fig. 2. The detailed configuration could be found in the supplementary materials.

\section{EXPERIMENTS}

\section{A. Dataset and Implementation Details}

Because EnlightenGAN has the unique ability to be trained with unpaired low/normal light images, we are enabled to collect a larger-scale unpaired training set, that covers diverse image qualities and contents. We assemble a mixture of 914 low light and 1016 normal light images from several datasets released in [47], [5] and also HDR sources [15], [25], without 

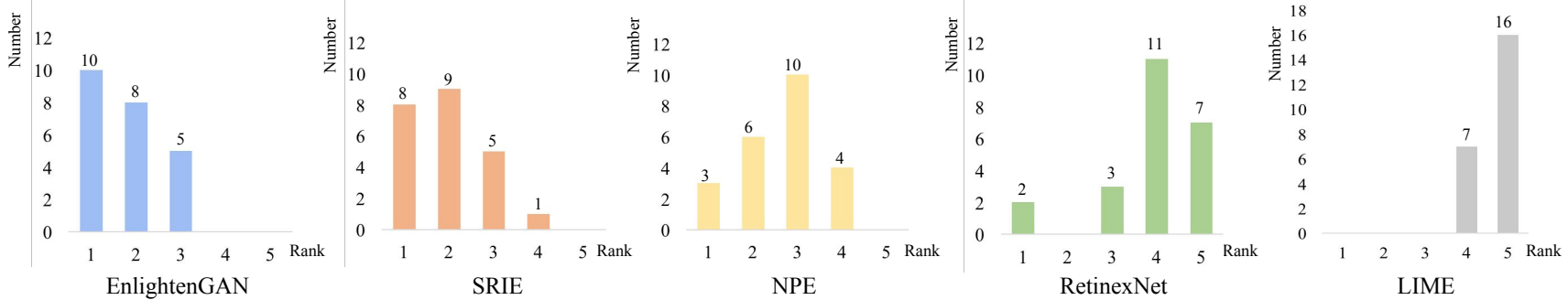

Fig. 5: The result of five methods in the human subjective evaluation. In each histogram, $x$-axis denotes the ranking index (1 $\sim 5,1$ represents the highest), and y-axis denotes the number of images in each ranking index. EnlightenGAN produces the most top-ranking images and gains the best performance with the smallest average ranking value.

the need to keep any pair. ${ }^{1}$ Manual inspection and selection are performed to remove images of medium brightness. All these photos are converted to PNG format and resized to $600 \times 400$ pixels. For testing images, we choose those standard ones used in previous works (NPE [19], LIME [21], MEF [48], DICM [49], VV, ${ }^{2}$ etc.).

EnlightenGAN is first trained from the scratch for 100 epochs with the learning rate of $1 \mathrm{e}-4$, followed by another 100 epochs with the learning rate linearly decayed to 0 . We use the Adam optimizer and the batch size is set to be 32 . Thanks to the lightweight design of one-path GAN without using cycle-consistency, the training time is much shorter than cycle based methods. The whole training process takes 3 hours on 3 Nvidia 1080Ti GPUs.

\section{B. Ablation Study}

To demonstrate the effectiveness of each component proposed in Sec. III, we conduct several ablation experiments. Specifically, we design two experiments by removing the components of local discriminator and attention mechanism, respectively. As shown in Fig. 3, the first row shows the input images. The second row shows the attention map of the input images, we can easily observe that the attention map gives a good guideline to the algorithm by which region should be enhanced more while others should be enhanced less. The third row shows the image produced by EnlightenGAN with only global discriminator to distinguish between low-light and normal-light images. The fourth row is the result produced by EnlightenGAN which does not adopt self-regularized attention mechanism and uses U-Net as the generator instead. The last row is produced by our proposed version of EnlightenGAN.

The enhanced results in the third row and the fourth row tend to contain local regions of severe color distortion or under-exposure, namely, the sky over the building in Fig.3(a), the roof region in Fig.3(b), the left blossom in Fig.3(c), the boundary of tree and bush in Fig.3(d), and the T-shirt in Fig.3(e). In contrast, the results of the full EnlightenGAN contain realistic color and thus more visually pleasing, which validates the effectiveness of the global-local discriminator

\footnotetext{
${ }^{1}$ The LOL dataset by [5] was a small paired dataset, but we did not use them as pairs for training. An exception is that, we hold out a subset of 50 low/normal light image pairs from LOL [5], as the validation set.

${ }^{2}$ https://sites.google.com/site/vonikakis/datasets
}

design and self-regularized attention mechanism. More images are in the supplementary materials.

\section{Comparison with State-of-the-Arts}

In this section we compare the performance of EnlightenGAN with current state-of-the-art methods. We conduct a list of experiments including visual quality comparison, human subjective review and no-referenced image quality assessment (IQA), which are elaborated on next.

1) Visual Quality Comparison: We first compare the visual quality of EnlightenGAN with several recent competing methods. Results are demonstrated in Fig. 4, where the first column shows the original low-light images, and the second to fifth columns are the images enhanced by: a vanilla CycleGAN [9] trained using our unpaired training set, RetinexNet [5], SRIE [20], LIME [21], NPE [19], LLNet [4], and CycleGAN [9]. The last column shows the results produced by EnlightenGAN.

We next zoom in on some details in the bounding boxes. LIME easily leads to over-exposure artifacts, which makes the results distorted and glaring with the some information missing. The results of SRIE and NPE are generally darker compared with others. CycleGAN and RetinexNet generate unsatisfactory visual results in terms of both brightness and naturalness. In contrast, EnlightenGAN successfully not only learns to enhance the dark area but also preserves the texture details and avoids over-exposure artifacts. More results are shown in the supplementary materials.

TABLE I: NIQE scores on the whole testing set (All) and each subset (MEF, LIME, NPE, VV, DICM) respectively. Smaller NIQE indicates more perceptually favored quality.

\begin{tabular}{|c|c|c|c|c|c|c|}
\hline Image set & MEF & LIME & NPE & VV & DICM & All \\
\hline \hline Input & 4.265 & 4.438 & 4.319 & 3.525 & 4.255 & 4.134 \\
\hline LLNet & 4.845 & 4.940 & 4.78 & 4.446 & 4.809 & 4.751 \\
\hline CycleGAN & 3.782 & 3.276 & 4.036 & 3.343 & 3.560 & 3.554 \\
\hline RetinexNet & 4.149 & 4.420 & 4.485 & 2.602 & 4.200 & 3.920 \\
\hline LIME & 3.720 & 4.155 & 4.268 & $\mathbf{2 . 4 8 9}$ & 3.846 & 3.629 \\
\hline SRIE & 3.475 & 3.788 & 3.986 & 2.850 & 3.899 & 3.650 \\
\hline NPE & 3.524 & 3.905 & $\mathbf{3 . 9 5 3}$ & 2.524 & 3.760 & 3.525 \\
\hline EnlightenGAN & $\mathbf{3 . 2 3 2}$ & $\mathbf{3 . 7 1 9}$ & 4.113 & 2.581 & $\mathbf{3 . 5 7 0}$ & $\mathbf{3 . 3 8 5}$ \\
\hline
\end{tabular}

2) No-Referenced Image Quality Assessment: We adopt Natural Image Quality Evaluator (NIQE) [50], a well-known no-reference image quality assessment for evaluating real 


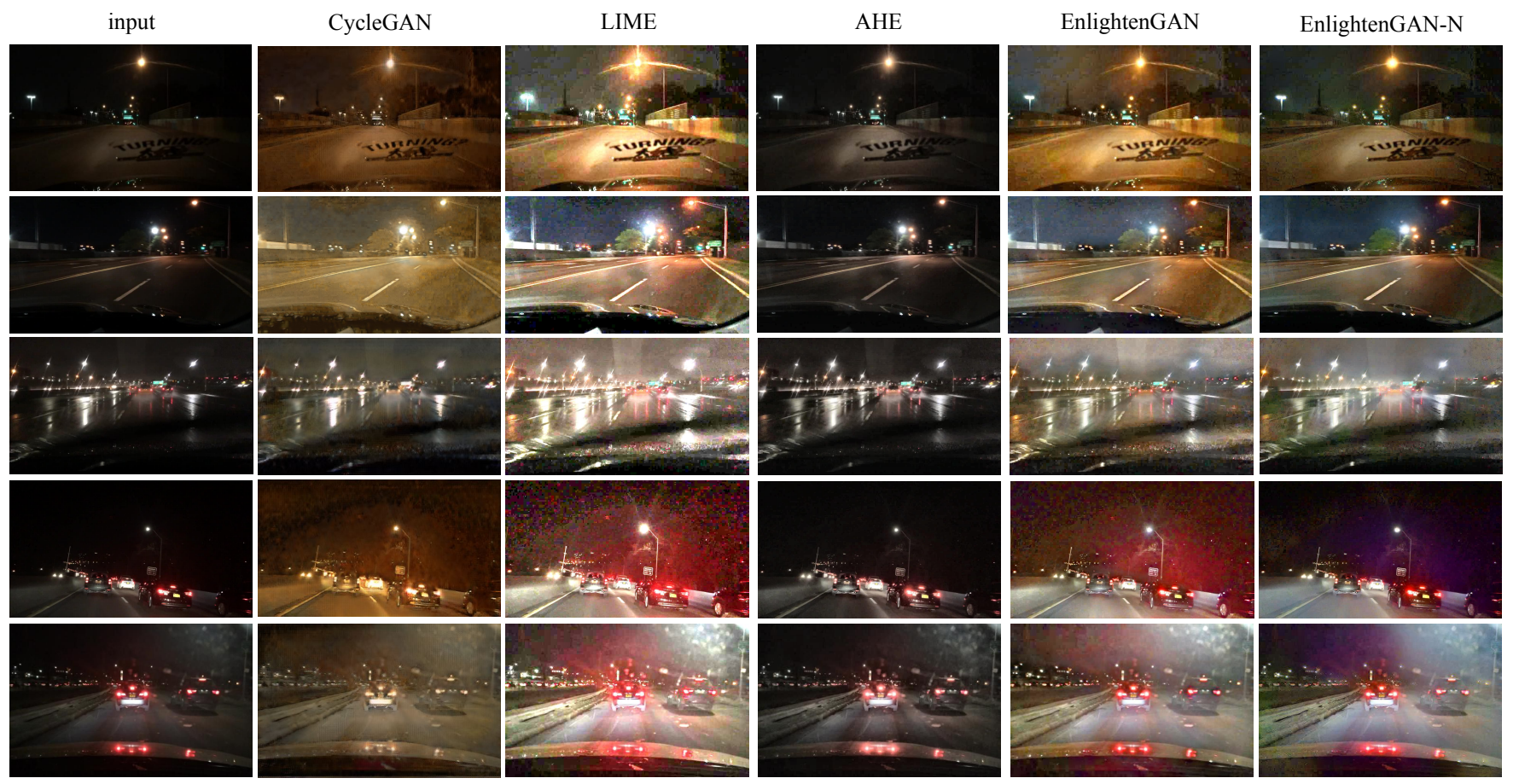

Fig. 6: Visual comparison of the results on the BBD-100k dataset [1]. EnlightenGAN-N is the domain-adapted version of EnlightenGAN, which generates the most visually pleasing results with noise suppressed. Please zoom in to see the details.

image restoration without ground-truth, to provide quantitative comparisons. The NIQE results on five publicly available image sets used by previous works (MEF, NPE, LIME, VV, and DICM) are reported in Table I: a lower NIQE value indicates better visual quality. EnlightenGAN wins on three out of five sets, and is the best in terms of overall averaged NIQE. This further endorses the superiority of EnlightenGAN over current state-of-the-art methods in generating high-quality visual results.

3) Human Subjective Evaluation: We conduct a human subjective study to compare the performance of EnlightenGAN and other methods. We randomly select 23 images from the testing set. For each image, it is first enhanced by five methods (LIME, RetinexNet, NPE, SRIE, and EnlightenGAN). We then ask 9 subjects to independently compare the five outputs in a pairwise manner. Specifically, each time a human subject is displayed with a pair of images randomly drawn from the five outputs, and is asked to evaluated which one has better quality. The human subjects are instructed to consider the: 1) whether the images contain visible noise; 2) whether the images contain over- or under-exposure artifacts; and 3) whether the images show nonrealistic color or texture distortions. Next, we fit a Bradley-Terry model [51] to estimate the numerical subjective scores so that the five methods can be ranked, using the exactly same routine as described in previous works [52]. As a result, each method is assigned with rank 1-5 on that image. We repeat the above for all 23 images.

Fig. 5 displays the five histograms, each of which depicts the rank distributions that a method receives on the 23 images. For example, EnlightGAN has been ranked the 1st (i.e., the highest subjective score) on 10 out of 23 images, the 2 nd for 8 images, and the 3 rd for 5 images. By comparing the five histograms, it is clear that EnlightenGAN produces the overall most favored results by human subjects, with an average ranking of 1.78 over 23 images. RetinexNet and LIME are not well scored, because of causing many over-exposures and sometimes amplifying the noise.

\section{Adaptation on Real-World Images}

Domain adaptation is an indispensable factor for real-world generalizable image enhancement. The unpaired training strategy of EnlightenGAN allows us to directly learn to enhance real-world low-light images from various domains, where there is no paired normal-light training data or even no normallight data from the same domain available. We conduct experiments using low-light images from a real-world driving dataset, Berkeley Deep Driving (BBD-100k) [1], to showcase this unique advantage of EnlightenGAN in practice.

We pick 950 night-time photos (selected by mean pixel intensity values smaller than 45) from the BBD-100k set as the low-light training images, plus 50 low-light images for hold-out testing. Those low-light images suffer from severe artifacts and high ISO noise. We then compare two EnlightenGAN versions trained on different normal-light image sets, including: 1) the pre-trained EnlightenGAN model as described in Sec. IV-A, without any adaptation for BBD100k; 2) EnlightenGAN-N: a domain-adapted version of EnlightenGAN, which uses BBD-100k low-light images from the BBD-100k dataset for training, while the normal-light images are still the high-quality ones from our unpaired dataset in Sec. IV-A. We also include a traditional method, Adaptive histogram equalization (AHE), and a pre-trained LIME model for comparison, and an unsupervised approach CycleGAN. 
As shown in Fig. 6, the results from LIME suffer from severe noise amplification and over-exposure artifacts, while AHE does not enhance the brightness enough. The unsupervised approach CycleGAN generate very low quality due to its unstability. The original EnlightenGAN also leads to noticeable artifacts on this unseen image domain. In comparison, EnlightenGAN-N produces the most visually pleasing results, striking an impressive balance between brightness and artifact/noise suppression. Thanks to the unpaired training, EnlightenGAN could be easily adapted into EnlightenGAN$\mathrm{N}$ without requiring any supervised/paired data in the new domain, which greatly facilitates its real-world generalization.

\section{E. Pre-Processing for Improving Classification}

Image enhancement as pre-processing for improving subsequent high-level vision tasks has recently received increasing attention [41], [53], [54], [55], with a number of benchmarking efforts [52], [56], [57], [58]. We investigate the impact of light enhancement on the extremely dark (ExDark) dataset [59], which was specifically built for the task of low-light image recognition. The classification results after light enhancement could be treated as an indirect measure on semantic information preservation, as [41], [52] suggested.

The ExDark dataset consists of 7,363 low-light images, including 3000 images in training set, 1800 images in validation set and 2563 images in testing set, annotated into 12 object classes. We use its testing set only, applying our pretrained EnlightenGAN as a pre-processing step, followed by passing through another ImageNet-pretrained ResNet-50 classifier. Neither domain adaption nor joint training is performed. The high-level task performance serves as a fixed semantic-aware metric for enhancement results.

In the low-light testing set, using EnlightenGAN as preprocessing improves the classification accuracy from $22.02 \%$ (top-1) and $39.46 \%$ (top-5), to $23.94 \%$ (top-1) and $40.92 \%$ (top-5) after enhancement. That supplies a side evidence that EnlightenGAN preserves semantic details, in addition to producing visually pleasing results. We also conduct experiment using LIME and AHE. LIME improves the accuracy to $23.32 \%$ (top-1) and $40.60 \%$ (top-5), while AHE obtains to $23.04 \%$ (top-1) and $40.37 \%$ (top-5).

\section{CONCLUSION}

In this paper, we address the low-light enhancement problem with a novel and flexible unsupervised framework. The proposed EnlightenGAN operates and generalizes well without any paired training data. The experimental results on various low light datasets show that our approach outperforms multiple state-of-the-art approaches under both subjective and objective metrics. Furthermore, we demonstrate that EnlightenGAN can be easily adapted on real noisy low-light images and yields visually pleasing enhanced images. Our future work will explore how to control and adjust the light enhancement levels based on user inputs in one unified model. Due to the complicacy of light enhancement, we also expect integrate algorithm with sensor innovations.

\section{REFERENCES}

[1] Fisher Yu, Wenqi Xian, Yingying Chen, Fangchen Liu, Mike Liao, Vashisht Madhavan, and Trevor Darrell. Bdd100k: A diverse driving video database with scalable annotation tooling. arXiv preprint arXiv:1805.04687, 2018. 1, 7

[2] Edwin H Land. The retinex theory of color vision. Scientific american, 237(6):108-129, 1977. 1, 2

[3] Stephen M Pizer, E Philip Amburn, John D Austin, Robert Cromartie, Ari Geselowitz, Trey Greer, Bart ter Haar Romeny, John B Zimmerman, and Karel Zuiderveld. Adaptive histogram equalization and its variations. Computer vision, graphics, and image processing, 39(3):355-368, 1987. 1, 2

[4] Kin Gwn Lore, Adedotun Akintayo, and Soumik Sarkar. Llnet: A deep autoencoder approach to natural low-light image enhancement. Pattern Recognition, 61:650-662, 2017. 1, 2, 6

[5] Chen Wei, Wenjing Wang, Wenhan Yang, and Jiaying Liu. Deep retinex decomposition for low-light enhancement. arXiv preprint arXiv:1808.04560, 2018. 1, 2, 5, 6

[6] Jiwon Kim, Jung Kwon Lee, and Kyoung Mu Lee. Accurate image super-resolution using very deep convolutional networks. In Proceedings of the IEEE conference on computer vision and pattern recognition, pages 1646-1654, 2016. 1

[7] Kai Zhang, Wangmeng Zuo, Yunjin Chen, Deyu Meng, and Lei Zhang. Beyond a gaussian denoiser: Residual learning of deep cnn for image denoising. IEEE Transactions on Image Processing, 26(7):3142-3155, 2017. 1

[8] Xin Tao, Hongyun Gao, Xiaoyong Shen, Jue Wang, and Jiaya Jia. Scalerecurrent network for deep image deblurring. In Proceedings of the IEEE Conference on Computer Vision and Pattern Recognition, pages 8174-8182, 2018. 1

[9] Jun-Yan Zhu, Taesung Park, Phillip Isola, and Alexei A Efros. Unpaired image-to-image translation using cycle-consistent adversarial networks. In $I C C V$, pages 2223-2232, 2017. 1, 3, 6

[10] Ming-Yu Liu, Thomas Breuel, and Jan Kautz. Unsupervised imageto-image translation networks. In Advances in Neural Information Processing Systems, pages 700-708, 2017. 1, 3

[11] Thekke Madam Nimisha, Kumar Sunil, and AN Rajagopalan. Unsupervised class-specific deblurring. In Proceedings of the European Conference on Computer Vision (ECCV), pages 353-369, 2018. 1

[12] Xun Huang, Ming-Yu Liu, Serge Belongie, and Jan Kautz. Multimodal unsupervised image-to-image translation. In Proceedings of the European Conference on Computer Vision (ECCV), pages 172-189, 2018. 1

[13] Yunjey Choi, Minje Choi, Munyoung Kim, Jung-Woo Ha, Sunghun Kim, and Jaegul Choo. Stargan: Unified generative adversarial networks for multi-domain image-to-image translation. In Proceedings of the IEEE Conference on Computer Vision and Pattern Recognition, pages 87898797, 2018. 1

[14] Judy Hoffman, Eric Tzeng, Taesung Park, Jun-Yan Zhu, Phillip Isola, Kate Saenko, Alexei A Efros, and Trevor Darrell. Cycada: Cycle-consistent adversarial domain adaptation. arXiv preprint arXiv:1711.03213, 2017. 1

[15] Nima Khademi Kalantari and Ravi Ramamoorthi. Deep high dynamic range imaging of dynamic scenes. ACM Trans. Graph, 36(4):144, 2017. $2,3,5$

[16] Chen Chen, Qifeng Chen, Jia Xu, and Vladlen Koltun. Learning to see in the dark. arXiv preprint arXiv:1805.01934, 2018. 2, 3

[17] Shangzhe Wu, Jiarui Xu, Yu-Wing Tai, and Chi-Keung Tang. Deep high dynamic range imaging with large foreground motions. In Proceedings of the European Conference on Computer Vision (ECCV), pages 117132, 2018. 2, 3

[18] Daniel J Jobson, Zia-ur Rahman, and Glenn A Woodell. A multiscale retinex for bridging the gap between color images and the human observation of scenes. IEEE Transactions on Image processing, 6(7):965-976, 1997. 2

[19] Shuhang Wang, Jin Zheng, Hai-Miao Hu, and Bo Li. Naturalness preserved enhancement algorithm for non-uniform illumination images. IEEE Transactions on Image Processing, 22(9):3538-3548, 2013. 2, 6

[20] Xueyang Fu, Delu Zeng, Yue Huang, Xiao-Ping Zhang, and Xinghao Ding. A weighted variational model for simultaneous reflectance and illumination estimation. In CVPR, pages 2782-2790, 2016. 2, 6

[21] Xiaojie Guo, Yu Li, and Haibin Ling. Lime: Low-light image enhancement via illumination map estimation. IEEE Transactions on Image Processing, 26(2):982-993, 2017. 2, 6 
[22] Xutong Ren, Mading Li, Wen-Huang Cheng, and Jiaying Liu. Joint enhancement and denoising method via sequential decomposition. In Circuits and Systems (ISCAS), 2018 IEEE International Symposium on pages 1-5. IEEE, 2018. 2

[23] Mading Li, Jiaying Liu, Wenhan Yang, Xiaoyan Sun, and Zongming Guo. Structure-revealing low-light image enhancement via robust retinex model. IEEE Transactions on Image Processing, 27(6):2828-2841, 2018. 2

[24] Michaël Gharbi, Jiawen Chen, Jonathan T Barron, Samuel W Hasinoff, and Frédo Durand. Deep bilateral learning for real-time image enhancement. ACM Transactions on Graphics (TOG), 36(4):118, 2017. 2

[25] Jianrui Cai, Shuhang Gu, and Lei Zhang. Learning a deep single image contrast enhancer from multi-exposure images. IEEE Transactions on Image Processing, 27(4):2049-2062, 2018. 3, 5

[26] Ian Goodfellow, Jean Pouget-Abadie, Mehdi Mirza, Bing Xu, David Warde-Farley, Sherjil Ozair, Aaron Courville, and Yoshua Bengio. Generative adversarial nets. In Advances in neural information processing systems, pages 2672-2680, 2014. 3

[27] Xinyu Gong, Shiyu Chang, Yifan Jiang, and Zhangyang Wang. Autogan: Neural architecture search for generative adversarial networks. In Proceedings of the IEEE International Conference on Computer Vision, pages $3224-3234,2019.3$

[28] Christian Ledig, Lucas Theis, Ferenc Huszár, Jose Caballero, Andrew Cunningham, Alejandro Acosta, Andrew P Aitken, Alykhan Tejani, Johannes Totz, Zehan Wang, et al. Photo-realistic single image superresolution using a generative adversarial network. In $C V P R$, volume 2 , page 4,2017 . 3, 4

[29] Shuai Yang, Zhangyang Wang, Zhaowen Wang, Ning Xu, Jiaying Liu, and Zongming Guo. Controllable artistic text style transfer via shapematching gan. In Proceedings of the IEEE International Conference on Computer Vision, pages 4442-4451, 2019. 3

[30] Shuai Yang, Zhangyang Wang, Jiaying Liu, and Zongming Guo. Deep plastic surgery: Robust and controllable image editing with humandrawn sketches. arXiv preprint arXiv:2001.02890, 2020. 3

[31] Rui Qian, Robby T Tan, Wenhan Yang, Jiajun Su, and Jiaying Liu. Attentive generative adversarial network for raindrop removal from a single image. In Proceedings of the IEEE Conference on Computer Vision and Pattern Recognition, pages 2482-2491, 2018. 3

[32] Runde Li, Jinshan Pan, Zechao Li, and Jinhui Tang. Single image dehazing via conditional generative adversarial network. methods, 3:24, 2018. 3

[33] Xitong Yang, Zheng $\mathrm{Xu}$, and Jiebo Luo. Towards perceptual image dehazing by physics-based disentanglement and adversarial training. In The Thirty-Second AAAI Conference on Artificial Intelligence (AAAI18), 2018. 3

[34] Yuan Yuan, Siyuan Liu, Jiawei Zhang, Yongbing Zhang, Chao Dong, and Liang Lin. Unsupervised image super-resolution using cycle-incycle generative adversarial networks. CVPR Workshops, 30:32, 2018. 3

[35] Yu-Sheng Chen, Yu-Ching Wang, Man-Hsin Kao, and Yung-Yu Chuang. Deep photo enhancer: Unpaired learning for image enhancement from photographs with gans. In Proceedings of the IEEE Conference on Computer Vision and Pattern Recognition, pages 6306-6314, 2018. 3

[36] Xin Jin, Zhibo Chen, Jianxin Lin, Zhikai Chen, and Wei Zhou. Unsupervised single image deraining with self-supervised constraints. arXiv preprint arXiv:1811.08575, 2018. 3

[37] Jiahui Yu, Zhe Lin, Jimei Yang, Xiaohui Shen, Xin Lu, and Thomas S Huang. Generative image inpainting with contextual attention. In Proceedings of the IEEE conference on computer vision and pattern recognition, pages 5505-5514, 2018. 3

[38] Alexia Jolicoeur-Martineau. The relativistic discriminator: a key element missing from standard gan. arXiv preprint arXiv:1807.00734, 2018. 3

[39] Xudong Mao, Qing Li, Haoran Xie, Raymond YK Lau, Zhen Wang, and Stephen Paul Smolley. Least squares generative adversarial networks. In Computer Vision (ICCV), 2017 IEEE International Conference on, pages 2813-2821. IEEE, 2017. 4

[40] Justin Johnson, Alexandre Alahi, and Li Fei-Fei. Perceptual losses for real-time style transfer and super-resolution. In European Conference on Computer Vision, pages 694-711. Springer, 2016. 4

[41] Orest Kupyn, Volodymyr Budzan, Mykola Mykhailych, Dmytro Mishkin, and Jiri Matas. Deblurgan: Blind motion deblurring using conditional adversarial networks. arXiv preprint arXiv:1711.07064, 2017. 4, 8

[42] Brandon RichardWebster, Samuel Anthony, and Walter Scheirer. Psyphy: a psychophysics driven evaluation framework for visual recognition. IEEE transactions on pattern analysis and machine intelligence, 2018. 4
[43] Dmitry Ulyanov, Andrea Vedaldi, and Victor S Lempitsky. Improved texture networks: Maximizing quality and diversity in feed-forward stylization and texture synthesis. In CVPR, volume 1, page 3, 2017. 5

[44] Olaf Ronneberger, Philipp Fischer, and Thomas Brox. U-net: Convolutional networks for biomedical image segmentation. In International Conference on Medical image computing and computer-assisted intervention, pages 234-241. Springer, 2015. 5

[45] Ding Liu, Bihan Wen, Xianming Liu, Zhangyang Wang, and Thomas S Huang. When image denoising meets high-level vision tasks: A deep learning approach. In IJCAI, 2018. 5

[46] Sergey Ioffe and Christian Szegedy. Batch normalization: Accelerating deep network training by reducing internal covariate shift. arXiv preprint arXiv:1502.03167, 2015. 5

[47] Duc-Tien Dang-Nguyen, Cecilia Pasquini, Valentina Conotter, and Giulia Boato. Raise: a raw images dataset for digital image forensics. In Proceedings of the 6th ACM Multimedia Systems Conference, pages 219-224. ACM, 2015. 5

[48] Kede Ma, Kai Zeng, and Zhou Wang. Perceptual quality assessment for multi-exposure image fusion. IEEE Transactions on Image Processing, 24(11):3345-3356, 2015. 6

[49] Chulwoo Lee, Chul Lee, and Chang-Su Kim. Contrast enhancement based on layered difference representation. In Image Processing (ICIP) 2012 19th IEEE International Conference on, pages 965-968. IEEE, 2012. 6

[50] Anish Mittal, Rajiv Soundararajan, and Alan C Bovik. Making a" completely blind" image quality analyzer. IEEE Signal Process. Lett., 20(3):209-212, 2013. 6

[51] Ralph Allan Bradley and Milton E Terry. Rank analysis of incomplete block designs: I. the method of paired comparisons. Biometrika, 39(3/4):324-345, 1952. 7

[52] Boyi Li, Wenqi Ren, Dengpan Fu, Dacheng Tao, Dan Feng, Wenjun Zeng, and Zhangyang Wang. Benchmarking single-image dehazing and beyond. IEEE Transactions on Image Processing, 28(1):492-505, 2019. 7, 8

[53] Boyi Li, Xiulian Peng, Zhangyang Wang, Jizheng Xu, and Dan Feng. Aod-net: All-in-one dehazing network. In Proceedings of the IEEE International Conference on Computer Vision, pages 4770-4778, 2017.

[54] Yu Liu, Guanlong Zhao, Boyuan Gong, Yang Li, Ritu Raj, Niraj Goel, Satya Kesav, Sandeep Gottimukkala, Zhangyang Wang, Wenqi Ren, et al. Improved techniques for learning to dehaze and beyond: A collective study. arXiv preprint arXiv:1807.00202, 2018. 8

[55] Orest Kupyn, Tetiana Martyniuk, Junru Wu, and Zhangyang Wang. Deblurgan-v2: Deblurring (orders-of-magnitude) faster and better. In Proceedings of the IEEE International Conference on Computer Vision, pages $8878-8887,2019.8$

[56] Ye Yuan, Wenhan Yang, Wenqi Ren, Jiaying Liu, Walter J Scheirer, and Zhangyang Wang. Ug2 track 2: A collective benchmark effort for evaluating and advancing image understanding in poor visibility environments. arXiv preprint arXiv:1904.04474, 2019. 8

[57] Siyuan Li, Iago Breno Araujo, Wenqi Ren, Zhangyang Wang, Eric K Tokuda, Roberto Hirata Junior, Roberto Cesar-Junior, Jiawan Zhang, Xiaojie Guo, and Xiaochun Cao. Single image deraining: A comprehensive benchmark analysis. arXiv preprint arXiv:1903.08558, 2019. 8

[58] Wenhan Yang, Ye Yuan, Wenqi Ren, Jiaying Liu, Walter J Scheirer, Zhangyang Wang, Taiheng Zhang, Qiaoyong Zhong, Di Xie, Shiliang $\mathrm{Pu}$, et al. Advancing image understanding in poor visibility environments: A collective benchmark study. IEEE Transactions on Image Processing, 29:5737-5752, 2020. 8

[59] Yuen Peng Loh and Chee Seng Chan. Getting to know low-light images with the exclusively dark dataset. Computer Vision and Image Understanding, 2018. 8 


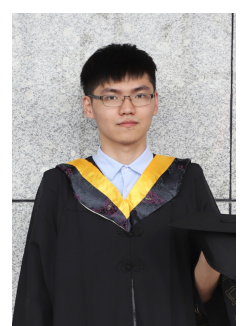

Yifan Jiang is currently a Ph.D. student at The University of Texas at Austin. He received his bachelor degree from the Huazhong University of Science and Technology in 2019. His research interests include computer vision and deep learning. Now he mainly works on the area of generative models and image enhancement.

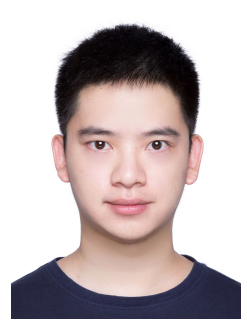

Xinyu Gong is currently a Ph.D. student of Electrical and Computer Engineering at The University of Texas at Austin. He receives his bachelor degree from University of Electronic Science and Technology of China in 2018. His research interests include AutoML and generative models.

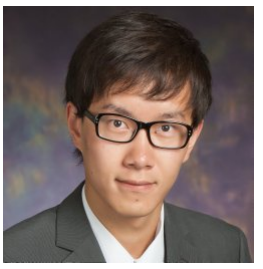

Ding Liu received the Ph.D. degree from the University of Illinois at Urbana- Champaign, USA, in 2018. He is currently a Research Scientist with Bytedance Inc., Mountain View, CA, USA. His research experience encompasses low-level vision problems, including image/video restoration and enhancement. He has broad research interests in the area of computer vision, image processing. and deep learning.

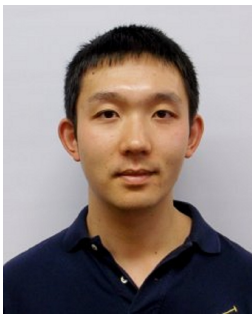

Yu Cheng is a researcher at Microsoft. Before that, he spent three years as a research staff member at IBM T.J. Watson Research Center. He got his Ph.D. from Northwestern University in 2015 and bachelors from Tsinghua University in 2010. His research is about deep learning in general, with specific interests in the deep generative model, model compression, and reinforcement learning. He is also interested in solving real-world problems of computer vision and natural language processing. He regularly serves on the program committees of top-tier AI conferences such as NIPS, ICML, ICLR, CVPR and ACL.

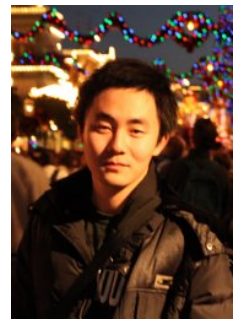

Chen Fang is a researcher at Bytedance Inc. Before that, he was a research scientist at Adobe Research. $\mathrm{He}$ got his Ph.D. from Dartmouth in 2015 and bachelors from Hunan University in 2010. His research interests include image understanding, image search, image editing, generative models

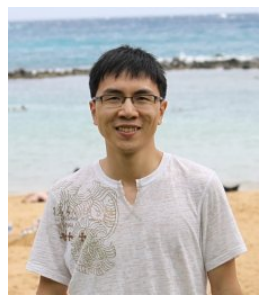

Xiaohui Shen is a Researcher at ByteDance AI Lab. Before that, he was a Senior Research Scientist at Adobe Research. He obtained his PhD degree from the Department of EECS at Northwestern University, and received the MS and BS degrees from the Department of Automation at Tsinghua University, China. His research interests include computer vision and deep learning.

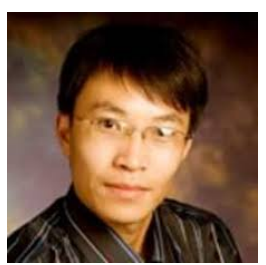

Jianchao Yang received the M.S. and Ph.D. degrees from the ECE Department, University of Illinois at Urbana-Champaign, under the supervision of Prof. Thomas Huang. He was a Research Scientist with Adobe Research. He is currently a Director with Bytedance Inc. He has authored over 80 technical papers over a wide variety of topics on top tier conferences and journals, with Google scholar citation over 12000 times. His research focuses on computer vision, deep learning, and image and video processing. He received the Best Student Paper award from ICCV 2010, the Classification Task Prize in PASCAL VOC 2009, first position for object localization using external data for ILSVRC ImageNet 2014, and third place in the WebVision Challenge 2017. He serves as the Workshop Chair of the ACM MM 2017.

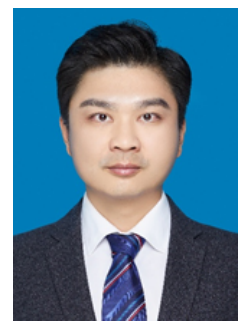

Pan Zhou is currently a full professor with Hubei Engineering Research Center on Big Data Security, School of Cyber Science and Engineering, Huazhong University of Science and Technology. He received his Ph.D. in the School of Electrical and Computer Engineering at the Georgia Institute of Technology (Georgia Tech) in 2011, Atlanta, USA. $\mathrm{He}$ was a senior technical member at Oracle Inc., from 2011 to 2013. His current research interests include security and privacy, big data analytics, machine learning, and information networks.

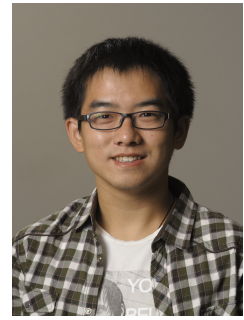

Zhangyang Wang is currently an Assistant Professor at The University of Texas at Austin. He was an Assistant Professor at Texas A\&M University, from 2017 to 2020. He received his Ph.D. degree the ECE Department, University of Illinois at Urbana-Champaign, under the supervision of Prof. Thomas Huang. Prof. Wang is broadly interested in the fields of machine learning, computer vision, optimization, and their interdisciplinary applications. His latest interests focus on automated machine learning (AutoML), learning-based optimization, machine learning robustness, and efficient deep learning. 